Pesq. Vet. Bras. 37(10):1125-1132, outubro 2017 DOI: $10.1590 / \mathrm{S} 0100-736 \mathrm{X} 2017001000014$

\title{
Caracterização clínica e histopatológica de bulbos oculares de cães e gatos (2005-2015) ${ }^{1}$
}

\begin{abstract}
Paula D. Galera ${ }^{2 *}$, Rosélia L.S. Araújo ${ }^{2}$, Fabiano J.F. de Sant’Ana ${ }^{2}$ e Márcio B. Castro ${ }^{2}$
ABSTRACT.- Galera P.D., Araújo R.L.S., Sant'Ana F.J.F. \& Castro M.B. 2017. [Clinical and histopathologic characterization of ocular bulbs in dogs and cats (2005-2015).] Caracterização clínica e histopatológica de bulbos oculares de cães e gatos (2005-2015). Pesquisa Veterinária Brasileira 37(10):1125-1132. Faculdade de Agronomia e Medicina Veterinária, Universidade de Brasília, Campus Universitário Darcy Ribeiro, Avenida L4 Norte, Asa Norte, Brasília, DF 70910-900, Brazil, E-mail: dra.paulagalera@gmail.com

This study aimed to carry out the clinical and histopathologic characterization of ocular bulbs from dogs and cats, surgical removed as clinical indication between 2005-2015. Hundred-one procedures were performed, 93 enucleations (92\%) and 8 exenterantions (8\%). The procedures were performed on 80 dogs ( $79 \%$ of cases) and 21 cats ( $21 \%$ of cases). The dogs underwent to surgery had ocular perfuration $(n=31,39 \%)$, glaucoma $(n=19,24 \%)$, ocular protrusion $(n=13,16 \%)$, diagnosis suggestive of neoplasia $(n=10,12,5 \%)$ and other $(n=3,2.97 \%)$. Among the clinical diagnosis cats comprise ocular perforation $(n=9,49 \%)$, glaucoma $(n=3,14.2 \%)$, microphthalmia $(n=3,14.2 \%)$, suggestive of neoplasia $(n=2,9.5 \%)$, ocular protrusion $(n=2,9.5 \%)$ and other $(n=2,9.5 \%)$. Twenty-seven samples of ocular bulbs were sent for histopathologic evaluation in Veterinary Pathology Laboratory, being 23 from dogs and four cats. Among the samples of dogs, seven exhibited histopathologic findings compatible with glaucoma, seven ocular perforations, six had intraocular neoplasias, two panoftalmites and condition of Phthisis bulbi. The ocular bulbs of cats received the diagnosis of two intraocular neoplasias, one microphthalmia and ocular perforation. It was found that the secondary glaucoma and ocular perforations are among the leading causes of enucleation in dogs, and associated with primary disorders such as keratoconjunctivitis sicca and uveitis. These disorders, unlike neoplasia, may have therapeutic success through early diagnosis and treatment, preventing blindness and removal of the eyeball.
\end{abstract}

INDEX TERMS: Ocular bulbs, dogs, cats, glaucoma, ocular perforation, ocular neoplasia, uveitis, keratoconjunctivitis sicca.

RESUMO.- Objetivou-se realizar a caracterização clínica e histopatológica de bulbos oculares de cães e gatos, removidos cirurgicamente por indicação clínica, no período entre 2005-2015. Foram realizados 101 procedimentos de remoção do bulbo ocular, 93 enucleações (92\%) e 8 exenterações (8\%). Os procedimentos foram realizados em 80 cães, ( $79 \%$ dos casos) e em 21 gatos ( $21 \%$ dos casos). Os cães submetidos à intervenção cirúrgica apresentavam perfurações oculares $(n=31,39 \%)$, glaucoma $(n=19,24 \%)$, protrusão ocular ( $\mathrm{n}=13,16 \%)$, diagnósticos sugestivos de neopla-

\footnotetext{
${ }^{1}$ Recebido em 13 de julho de 2016.

Aceito para publicação em 29 de novembro de 2016.

${ }^{2}$ Faculdade de Agronomia e Medicina Veterinária (FAV), Universidade de Brasília (UnB), Brasília, DF 70910-900, Brasil. *Autor para correspondência: dra.paulagalera@gmail.com
}

sia $(n=10,12,5 \%)$ e outros $(n=3,2,97 \%)$. Dentre os gatos os diagnósticos clínicos compreenderam perfuração ocular $(\mathrm{n}=9,49 \%)$, glaucoma $(\mathrm{n}=3,14,2 \%)$, microftalmia $(\mathrm{n}=3$, $14,2 \%)$, sugestivo de neoplasia $(n=2,9,5 \%)$, protrusão ocular $(n=2,9,5 \%)$ e outros $(n=2,9,5 \%)$. Vinte e sete amostras de bulbos oculares foram submetidas para avaliação histopatológica, sendo 23 provenientes de cães e quatro de gatos. Dentre as amostras de origem canina, sete exibiram achados histopatológicos compatíveis com glaucoma, sete perfurações oculares, seis neoplasias intraoculares, duas panoftalmites e um quadro de Phthisis bulbi. Nos bulbos oculares de gatos foram diagnosticadas duas neoplasias intraoculares, uma microftalmia e uma perfuração ocular. Constatou-se que o glaucoma secundário, as neoplasias e as perfurações oculares estão entre as principais causas de enucleação em cães, e associadas a alterações primárias 
como a ceratoconjuntivite seca e as uveítes. Estas afecções, diferentemente das neoplasias, podem ter êxito terapêutico mediante diagnóstico e tratamento precoces, prevenindo a cegueira e a remoção do bulbo ocular.

TERMOS DE INDEXAÇÃO: Bulbos oculares, cães, gatos, glaucoma, perfuração ocular, neoplasia ocular, uveíte, ceratoconjuntivite seca.

\section{INTRODUÇÃO}

O desenvolvimento da área de oftalmologia veterinária requer precisão diagnóstica e um melhor entendimento das alterações oculares. A remoção cirúrgica do bulbo ocular compreende importante opção terapêutica em casos de afecções dolorosas e de cegueira irreversível, a exemplo de glaucomas incontroláveis, neoplasias, perfurações e traumas oculares (Dubielzig et al. 2010). A enucleação (remoção do bulbo ocular), exenteração (remoção do bulbo ocular, conteúdo orbitário e pálpebras) e a evisceração (remoção do contéudo intraocular) são opções cirúrgicas com indicações específicas (Slatter 2008). A enucleação é preconizada em casos de neoplasia intraocular, traumas oculares perfurantes (com ruptura e perda de contéudo intraocular), glaucomas não responsivos a terapia, endoftalmites e panoftalmites intratáveis. A exenteração comumente é realizada em casos de neoplasia orbitária e aquelas que ultrapassem o bulbo ocular (Slatter 2008).

A avaliação anatomopatológica auxilia na compreensão dos processos que levam a perda do olho (Turner 2010). A histopatologia possibilita tipificar lesões neoplásicas em primárias ou secundárias (metastáticas), diferenciar e identificar alterações que levam ao glaucoma, bem como um possível acometimento ocular por doenças sistêmicas (Massa et al. 2002, Dubielzig et al. 2010).

Este estudo teve como objetivo caracterizar as alterações clínicas e histopatológicas de bulbos oculares enucleados e exenterados de cães e gatos provenientes do Serviço de Oftalmologia Veterinária do Hospital Veterinário da Faculdade de Agronomia e Medicina Veterinária da Universidade de Brasília (FAV-UnB), entre 2005 e 2015.

\section{MATERIAL E MÉTODOS}

Foram revisados os registros clínicos de cães e gatos atendidos pelo Serviço de Oftalmologia Veterinária do Hospital Veterinário (FAV-UnB) , entre maio de 2005 e dezembro de 2015. Foram incluídos no estudo os dados de todos os animais encaminhados para procedimentos cirúrgicos de enucleações ou exenterações. A partir da análise dos prontuários médicos foram coletadas as informações clínicas (diagnóstico, sexo, raça, idade) e encaminhamento cirúrgico.

Os dados histopatológicos foram obtidos por meio da revisão dos registros das amostras processadas pelo Laboratório de Patologia Veterinária (LPV-UnB), no mesmo período. Foram compiladas informações epidemiológicas (raça, sexo, idade), sinais clínicos e alterações macroscópicas descritas. Os bulbos oculares submetidos ao LPV receberam injeção de formol a 10\% na câmara vítrea no momento do recebimento da amostra. Em seguida, foram inicialmente mantidos em solução de formol a 10\% por 24 horas e posteriormente fixados em solução de Davidson (formaldeído, etanol, ácido acético) (Dubielzig et al. 2010), por mais 24 horas. 0 corte do bulbo ocular foi realizado perpendicular à artéria ciliar e paralelo ao nervo óptico. Posteriormente, as amostras foram rotineiramente processadas para exame histopatológico, incluídas em parafina, cortadas a $5 \mu \mathrm{m}$ de espessura e coradas pela hematoxilina-eosina (HE). As lesões histopatológicas de todos os casos selecionados foram identificadas e reavaliadas as alterações oculares na córnea, câmaras anterior e posterior, úvea, câmara vítrea, lente e retina.

A confirmação histopatológica de melanoma uveal em um cão foi realizada por meio da coloração imuno-histoquímica, a qual foi empregada em cortes histológicos de $4 \mu \mathrm{m}$ de espessura, dispostos em lâminas previamente tratadas com o aderente poli-D-lisina. A desparafinação foi feita em xilol seguida da hidratação em

solução aquosa de álcool etílico e lavagem com água. Após o bloqueio da peroxidase endógena com água oxigenada a 3\% por dez minutos, os cortes histológicos foram dispostos em solução de ácido cítrico a $0,01 \mathrm{M}, \mathrm{pH} 6$ e submetidos à temperatura de $121^{\circ} \mathrm{C}$ em microondas por 15 minutos para a recuperação antigênica. Após 20 minutos, incubou-se o anticorpo primário Melan-A (Clone A103; Dako Corporation S.A.) na titulação de 1:100 por 18 horas a $4^{\circ} \mathrm{C}$ em câmara úmida. Após a lavagem das lâminas em solução salina tamponada, foram adicionados os reagentes secundário (Biotinilado anti-mouse Ba 2000 - Vector) e terciário (ABC PK 6100 - Vector), incubando-os por 60 e 45 minutos, respectivamente. A reação foi revelada com DAB (Sigma Co., St Louis, EUA) por cinco minutos e contracorada com hematoxilina de Harris na diluição 10:1 (Salvio \& Marques 2006).

Este estudo foi aprovado pelo Comitê de Ética de Uso Animal (CEUA-UnB)(protocolo 60101/2016), atendendo às normas para experimentação animal da Association for Research in Vision and Ophthalmology (ARVO).

\section{RESULTADOS}

\section{Análise clínica}

Foram realizados 2.495 atendimentos de casos novos pelo Serviço de Oftalmologia Veterinária durante o período estudado, dos quais $97(3,8 \%)$ animais foram diagnosticados com afecções que levaram a remoção do bulbo ocular, sendo 84 enucleações unilaterais, quatro enucleações bilaterais (somando oito bulbos) e nove exenterações, totalizando 101 bulbos oculares removidos. Os procedimentos ocorreram predominantemente em cães, representando 79\% (80/101) dos casos, e os gatos constituíram 21\% (21/101). As enucleações bilaterais foram realizadas em cães diagnosticados com glaucoma. As exenterações ocorreram em animais com diagnósticos sugestivos de neoplasia ocular (sete cães e um gato) e trauma ocular (um gato). Os diagnósticos e informações clínicas dos cães e gatos enucleados são apresentados nos Quadros 1 e 2, respectivamente.

As perfurações oculares identificadas decorreram de lesões traumáticas provenientes de mordeduras, arranhaduras, perfurações por corpos estranhos e complicações de quadros de ceratoconjuntivite seca. Dezenove cães e três gatos foram enucleados como opção terapêutica ao glaucoma.

\section{Análise histopatológica}

Das 101 enucleações e exenterações realizadas em cães e gatos, 27 amostras de bulbos oculares foram enviadas para a avaliação histopatológica no LPV-UnB. Os principais achados histopatológicos visualizados na córnea, câmaras anterior e posterior, úvea, câmara vítrea, lente e retina dos 
Quadro 1. Dados clínicos de bulbos oculares de cães removidos cirurgicamente entre 2005 e 2015

\begin{tabular}{|c|c|c|c|c|c|}
\hline Diagnóstico Clínico & Machos & Fêmeas & NI* & Raças & Faixa etária (anos) \\
\hline Perfuração Ocular (n=13) & 18 & 13 & $?$ & $\begin{array}{l}\text { Poodle }(n=6) \text {, Shih tzu }(n=5) \text {, York } \\
\text { Shire }(n=4), S R D(n=4) \text {, Pinscher }(n=1) \text {, } \\
\text { Boxer }(n=1) \text {, Lhasa Apso }(n=1) \text {, Fila } \\
(n=1) \text {, Pug }(n=1) \text {, Beagle }(n=1) \text {, Fox } \\
\text { Paulistinha }(n=1) \text {, Mastin Nap. }(n=1) \text {, } \\
\text { Maltês }(n=1) \text {, Dachshund }(n=1)\end{array}$ & $1-16$ \\
\hline Glaucoma ( $n=19)$ & 8 & 8 & 3 & $\begin{array}{l}\text { SRD }(n=5) \text {, Poodle }(n=4) \text {, Pinscher }(n=1) \text {, } \\
\text { Cocker Spaniel }(n=1) \text {, Lhasa Apso }(n=1) \text {, } \\
\text { Chow Chow }(n=1) \text {, Pastor Alemão }(n=1) \text {, } \\
\text { Bassed Hound }(n=1) \text {, Boxer }(n=1), \\
\text { Bulldog Francês }(n=1) \text {, Pitbull }(n=1)\end{array}$ & $2-13$ \\
\hline Protusão ocular (n=13) & 7 & 3 & 3 & $\begin{array}{l}\text { Poodle }(n=4) \text {, Shih Tzu }(n=3) \text {, Pinscher } \\
(n=2) \text {, York Shire }(n=1) \text {, Pug }(n=1) \\
\text { SRD }(n=1) \text {, Não informada }(n=1)\end{array}$ & $0-12$ \\
\hline Neoplasia ocular $(n=10)$ & 3 & 7 & - & $\begin{array}{l}\text { Boxer }(\mathrm{n}=2) \text {, York Shire }(\mathrm{n}=1) \text {, Cocker } \\
\text { Spaniel }(\mathrm{n}=1) \text {, Chow Chow }(\mathrm{n}=1) \\
\text { Pastor Alemão }(\mathrm{n}=1) \text {, Lhasa Apso } \\
(\mathrm{n}=1) \text {, Fila }(\mathrm{n}=1) \text {, Pug }(\mathrm{n}=1) \\
\text { SRD }(\mathrm{n}=1) \text {, Não informada(n=1) }\end{array}$ & $3-11$ \\
\hline Phthisis bulbi $(\mathrm{n}=1)$ & 1 & $?$ & - & Poodle $(n=1)$ & 14 \\
\hline Abscesso Intraocular (n=1) & ? & 1 & ? & Shih Tzu $(n=1)$ & 4 \\
\hline Não informado (n=1) & 1 & - & - & Poodle $(n=1)$ & 6 \\
\hline Total $(n=76)$ & 38 & 32 & 36 & - & - \\
\hline
\end{tabular}

Apresentação de dados epidemiológicos e diagnósticos clínicos de cães enucleados entre Maio/2015 e Dezembro/2015. * NI = sexo não informado.

Quadro 2. Dados clínicos de bulbos oculares de gatos removidos cirurgicamente entre 2005 e 2015

\begin{tabular}{lccccc}
\hline Diagnóstico Clínico & Machos & Fêmeas & NI* & Raças & Faixa etária (anos) \\
\hline Perfuração Ocular (n=9) & 4 & 4 & 1 & SRD (n=6), Siamês (n=2),Persa (n=1) & 1 \\
Glaucoma (n=3) & 1 & 2 & - & SRD (n=2), Persa (n=1) & $1-18$ \\
Microftalmia (n=3) & 1 & 2 & - & -6 & $1-6$ \\
Neoplasia ocular (n=2) & 2 & - & - & SRD & $1-9$ \\
Protusão ocular (n=2) & 1 & 1 & - & SRD & 23 (meses) \\
Phthisis bulbi $(\mathrm{n}=1)$ & 1 & $?$ & - & SRD & 3 \\
Trauma Ocular (n=1) & 1 & $?$ & - & SRD & 1 (mês) \\
Total (n=21) & 11 & 9 & 1 & - & -
\end{tabular}

Apresentação de dados epidemiológicos e diagnósticos clínicos de gatos enucleados entre 2005 e 2015. * NI = sexo não informado.

bulbos oculares de cães foram resumidos no Quadro 3, e de gatos no Quadro 4.

Os bulbos oculares de cães totalizaram 23 (85\%) amostras e os de gatos, quatro amostras (15\%). Olhos de sete cães apresentaram achados histopatológicos compatíveis com glaucoma, sete perfurações oculares, seguidos por seis apresentando neoplasia intraocular, dois casos de afecções inflamatórias, além de um quadro de Phthisis bulbi. Nos bulbos oculares de gatos foram diagnosticadas duas neoplasias intraoculares, uma microftalmia e uma perfuração ocular.

Sete bulbos oculares de cães apresentaram achados compatíveis com glaucoma, destes seis foram diagnosticados como glaucoma secundário e em um bulbo ocular de uma cadela, da raça Bulldog Francês identificou-se uma displasia de ligamento pectinado (Fig.1A) associado à deposição de pigmento na região límbica, achados patognomônicos de glaucoma primário. Em todos os casos visualizou-se a oclusão do ângulo iridocorneal. Membranas fibrovasculares (Fig.1B), descolamentos de retina (Fig.1C) e cistos uveais foram achados frequentes.

Sete amostras de bulbos oculares de cães apresentando perfurações oculares foram examinadas, nestas foram visu- alizadas rupturas e prolapsos de estruturas intraoculares, assim como extravasamento de conteúdo intraocular e exsudatos (Fig.1D).

Dentre as neoplasias oculares, verificou-se o diagnóstico de dois carcinomas e um adenocarcinoma iridociliar em três cães. Em ambos os casos, observou-se que as células neoplásicas, ocasionalmente formando rosetas e pseudorosetas, expandiram o corpo ciliar e a íris levando a oclusão do ângulo de filtração iridociliar. Em um caso de carcinoma iridociliar, a proliferação neoplásica apresentou continuidade com o epitélio do corpo ciliar substituindo o tecido uveal adjacente e expandindo as câmaras anterior e posterior (Fig.1E), levando ao rompimento corneal.

Um hemangiossarcoma intraocular foi diagnosticado em um cão. Histologicamente observou-se uma massa neoplásica formando feixes e espaços vasculares por toda a córnea causando uma extensa ulceração corneal.

Um cão da raça Cocker Spaniel apresentou melanoma uveal, o qual foi confirmado pela técnica de imuno-histoquímica com o emprego do anticorpo Melan-A. Dentre as alterações histopatológicas visualizadas estão aglomerados de células neoplásicas, com o citoplasma preenchido por 
Quadro 3. Avaliação histopatológica dos bulbos oculares de cães $(\mathrm{n}=23)$ removidos cirurgicamente entre 2005 e 2015

\begin{tabular}{|c|c|c|c|c|c|c|c|c|c|}
\hline & \multirow{2}{*}{$\begin{array}{c}\text { Achados } \\
\text { Histopatológicos }\end{array}$} & \multicolumn{8}{|c|}{ Diagnósticos } \\
\hline & & \multicolumn{8}{|c|}{ Neoplasia ocular $(n=6)$} \\
\hline \multirow[t]{4}{*}{ Córnea } & Úlcera corneal & 2 & 7 & 1 & 1 & 1 & 1 & - & \\
\hline & Hiperplasia epitelial & 4 & - & - & - & 1 & 1 & - & - \\
\hline & Edema & 5 & 4 & 1 & 1 & 1 & - & - & 1 \\
\hline & Descemetocele & - & 2 & - & - & - & - & - & - \\
\hline Câmara anterior & Hifema & 3 & 2 & 1 & - & - & - & - & 1 \\
\hline \multirow{2}{*}{ e posterior } & Infiltrado neutrofílico & 3 & 5 & 1 & - & 1 & - & - & 1 \\
\hline & Infiltrado linfoplasmocítico & 1 & 2 & - & - & 1 & - & - & - \\
\hline \multirow[t]{2}{*}{ Úvea } & Oclusão de ângulo iridocorneal & 7 & 1 & 1 & 1 & 1 & 1 & 1 & - \\
\hline & Sinéquia anterior & 2 & 2 & 1 & - & 1 & 1 & - & 1 \\
\hline \multirow[t]{2}{*}{ Lente } & Catarata & 2 & - & - & - & 1 & - & - & - \\
\hline & Ruptura de cápsula & 2 & 1 & - & - & 1 & - & - & 1 \\
\hline \multirow[t]{2}{*}{ Retina } & Descolamento6 & 6 & 1 & 1 & 1 & - & 1 & 1 & - \\
\hline & Atrofia & 7 & - & - & - & 1 & - & 1 & - \\
\hline
\end{tabular}

Apresentação dos achados histopatológicos de cães subdivididos de acordo com as estruturas oculares de sua localização.

Quadro 4. Avaliação histopatológica dos bulbos oculares de gatos $(n=4)$ removidos cirurgicamente entre 2005 e 2015

\begin{tabular}{|c|c|c|c|c|c|}
\hline & \multirow{2}{*}{$\begin{array}{c}\text { Achados } \\
\text { Histopatológicos }\end{array}$} & \multicolumn{4}{|c|}{ Diagnósticos } \\
\hline & & $\begin{array}{l}\text { Carcinoma de Células } \\
\text { Escamosas }(n=2)\end{array}$ & $\begin{array}{c}\text { Perfuração } \\
\text { Ocular }(n=1)\end{array}$ & $\begin{array}{l}\text { Microftalmia } \\
\quad(n=1)\end{array}$ & Total \\
\hline \multirow[t]{4}{*}{ Córnea } & Úlcera corneal & - & 1 & - & 1 \\
\hline & Edema & 1 & 1 & - & 2 \\
\hline & Neovascularização superficial & - & 1 & - & 1 \\
\hline & Neovascularização profunda & - & 1 & - & 1 \\
\hline \multirow[t]{2}{*}{ Câmara anterior } & Hifema & - & 1 & - & 1 \\
\hline & Infiltrado linfoplasmocítico & 1 & 1 & - & 2 \\
\hline \multirow[t]{2}{*}{ Úvea } & Oclusão de ângulo iridocorneal & 2 & 1 & - & 3 \\
\hline & Membrana fibrovascular & - & 1 & - & 1 \\
\hline
\end{tabular}

grânulos amarronzados, expandindo as estruturas uveais. Em dois bulbos oculares de cães diagnosticou-se quadros de panoftalmites, em ambos os casos foram visualizados infiltrados inflamatórios acentuados, neovascularizações superficiais e profundas, e sinéquias (Fig.1F). Em um dos casos identificou-se o deslocamento anterior da lente. 0 diagnóstico de Phthisis bulbi em uma amostra de cão foi confirmado diante da observação da atrofia das estruturas oculares (córnea, íris, corpo ciliar, coroide, retina).

Dois carcinomas de células escamosas (CCE) foram diagnosticados em dois gatos. Nos CCEs os achados histológicos observados nos tecidos perioculares dos felinos foram células epiteliais neoplásicas e lamelas concêntricas compostas por material eosinofílico, caracterizando pérolas córneas, circundadas por infiltrado linfocítico acentuado. Em ambos os casos a proliferação neoplásica originava-se no tecido periocular adjacente à córnea, aderindo-se e invadindo a mesma; as amostras foram oriundas de exenterações. Um caso de microftalmia foi diagnosticado em um gato macho, que demonstrou ausência do desenvolvimento das estruturas intraoculares.

\section{DISCUSSÃO}

Verificou-se que as remoções de bulbos oculares, compreendidas como opção a tratamentos não responsivos ou indicações primárias de neoplasias (Labelle \& Labelle 2013) perfizeram 3,8\% (97 casos) da casuística avaliada. Este estudo relacionou os diagnósticos clínicos dos animais submetidos à remoção cirúrgica do bulbo diretamente com seus respectivos diagnósticos histopatológicos; os bulbos analisados foram exclusivamente provenientes de enucleações e exenterações realizadas pelo serviço supracitado. Em alguns estudos no Brasil foi realizada a análise patológica de afecções oculares em animais domésticos (cães, gatos, ruminantes e equinos), tais como neoplasias oculares e glaucoma (Martins \& Barros 2014, Hesse et al. 2015), no entanto, em um dos estudos foram avaliadas afecções perioculares e intraoculares, na outra pesquisa as amos- 

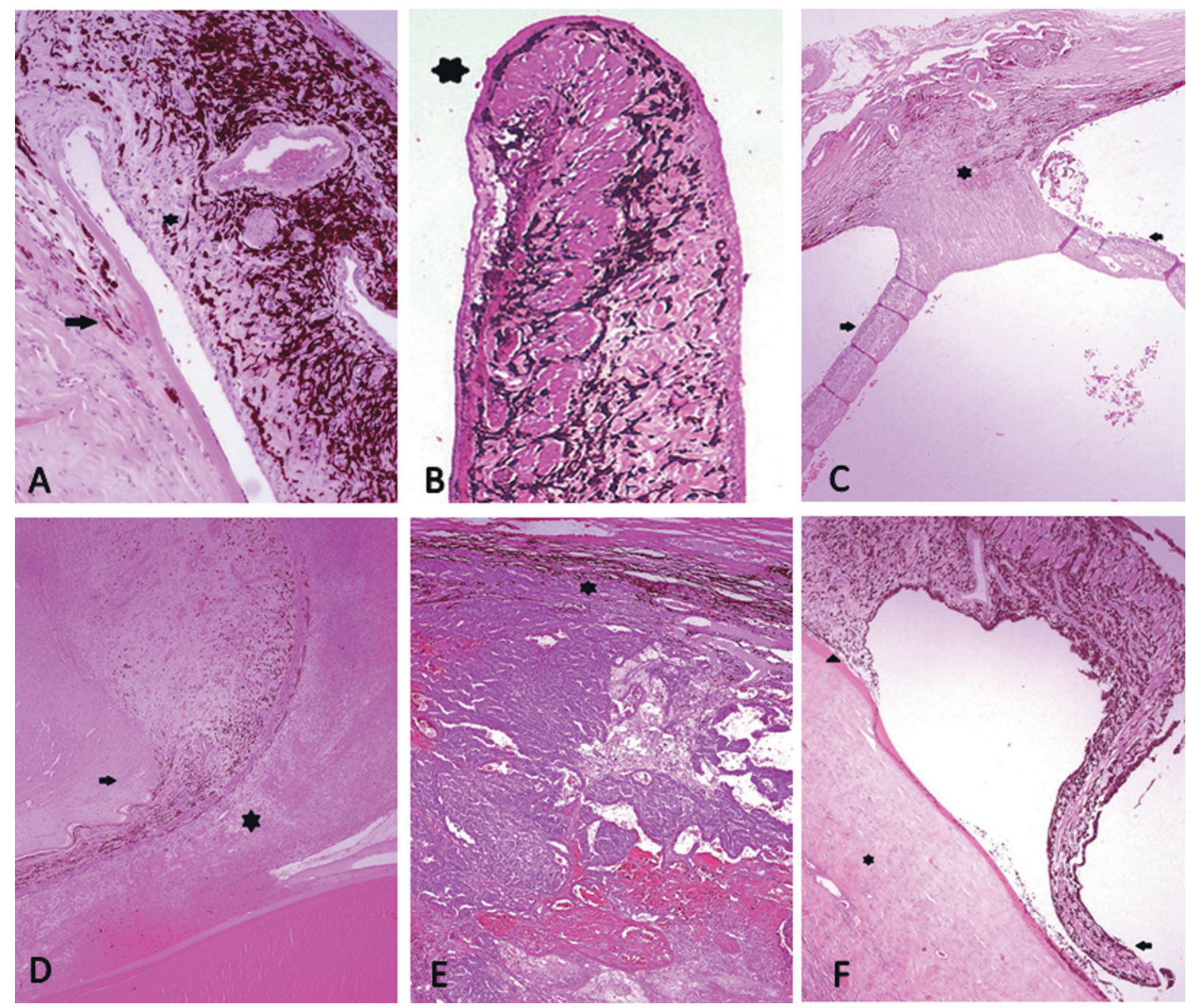

Fig.1. (A) Cão, fêmea, Bulldog Inglês. Nota-se displasia do ligamento pectinado (segmento do estroma uveal estendendo-se da base da íris a membrana de Descemet) (seta), achado característico de glaucoma primário. HE, 10x. (B) Membrana fibrovascular envolvendo e espessando a íris, observa-se a eversão da porção pupilar levando ao entrópio uveal (*). HE, 4x. (C) Cão, fêmea, Poodle. Nota-se o descolamento de retina (setas), a retina manteve-se aderida apenas ao disco óptico . HE, 4x. (D) Cão, macho, Lhasa Apso. Visualizar acentuado exsudato inflamatório (*) e prolapso de íris (seta) em um caso de perfuração ocular com extravasamento de conteúdo intraocular. HE, 4x. (E) Cão, fêmea, Fila Brasileiro. Carcinoma iridociliar. Observa-se proliferação neoplásica expandindo-se a partir do corpo ciliar (*). HE, 4x. (F) Adesão da íris em dois pontos da córnea (*) formando sinéquia anterior (seta) e sinéquia anterior periférica (ponta de seta). HE, $4 \mathrm{x}$.

tras avaliadas eram provenientes tanto de uma instituição universitária como de estabelecimentos particulares. Dessa forma, não é possível avaliar o impacto do número de enucleações e exenterações relacionados neste estudo, em relação a outros serviços de oftalmologia do país. Dada a conduta adotada para enucleações, que permitia a opção do proprietário pela análise histopatológica até o ano de 2012, à exceção dos casos de neoplasias, apenas 27 bulbos oculares foram enviados para a análise histopatológica. Em 2012, vinculou-se a enucleação à avaliação histológica.

A avaliação clínica constatou que as perfurações oculares foram a principal causa das enucleações realizadas em cães e gatos atendidos pelo Serviço de Oftalmologia Veterinária do Hospital Veterinário (FAV-UnB). Quarenta animais (31 cães, 9 gatos) foram clinicamente diagnosticados com esta afecção, considerada comum na rotina oftálmica (Paulsen \& Kass 2012). Tais perfurações foram predominantemente associadas a complicações de quadros de ceratoconjuntivite seca (CCS) e lesões traumáticas proveniente de mordeduras. Cães da raça Poodle e braquicefálicos como Shih Tzu, Pug e Lhasa Apso, incriminados como predisponentes às alterações do filme lacrimal (Slatter 2008), foram os mais acometidos por perfurações oculares decorrentes de complicações da CCS, tida como uma afecção inflamatória progressiva da córnea e da conjuntiva causada por deficiência na produção lacrimal (Grahn \& Sandmeyer 2007, Slatter 2008, Westermeyer et al. 2009, Matheis et al. 2012, Barachetti et al. 2015). A ruptura das estruturas corneais (epitélio, estroma, membrana de Descemet e endotélio), edema corneal, descolamento de retina, neovascularizações superficiais e profundas associadas a infiltrados inflamatórios foram comumente encontrados, evidenciando a cronicidade das lesões (Slatter 2008, Mc Gavin \& Zachary 2009, Dubielzig et al. 2010).

Nas amostras com perfurações oculares decorrentes de lesões traumáticas (dois cães e um gato), a visualização de prolapsos das estruturas intraoculares rupturas da membrana de Descemet, cicatrizes corneais substituindo 
o estroma corneal e aprisionando o tecido uveal, infiltrados inflamatórios neutrofilícos acentuados e hemorragias foram achados frequentes, geralmente observados em lesões perfurantes (Slatter 2008, McGavin \& Zachary 2009). No entanto, não é possível determinar se essas alterações antecederam a lesão traumática, conforme descrito previamente (Dubielzig et al. 2010).

Verificou-se que o glaucoma foi a segunda maior causa de enucleação de cães e gatos atendidos no referido serviço. Os glaucomas são caracterizados como um grupo de doenças oftálmicas geralmente associadas com o aumento da pressão intraocular, normalmente resultante de drenagem prejudicada do humor aquoso levando a alterações na fisiologia do nervo óptico, disfunção das células ganglionares da retina e cegueira progressiva (Tombran-Tink et al. 2008, Esson et al. 2009, Dubielzig et al. 2010, Yanoff \& Ducker 2014).

O glaucoma pode ser classificado como primário ou secundário (Dubielzig et al. 2010, Strom et al. 2011), apresentando ângulo aberto, em que humor aquoso tem acesso livre à malha trabecular ou glaucoma de ângulo fechado, em que o acesso do humor aquoso a malha trabecular está obstruído (Yanoff \& Ducker 2014). 0 glaucoma primário pode ser bilateral e hereditário (Reilly et al. 2005), geralmente associado à goniodisgenesia (Reilly et al. 2005, Gellat et al. 2007, Grahn \& Peiffer 2007), uma alteração congênita, em que há má formação do ângulo de filtração iridocorneal, como falha na atrofia do mesênquima embrionário (hipoplasia trabecular) ou displasia do ligamento pectinado (Reilly et al. 2005, Dubielzig et al. 2010, Turner 2010). Histologicamente, a displasia do ligamento pectinado apresenta-se como um segmento do estroma uveal (iridiano) estendendo-se da base da íris à membrana de Descemet (Reilly et al. 2005, Dubielzig et al. 2010). Neste estudo, este achado foi observado em um cão da raça Bulldog Francês, raça predisposta ao desenvolvimento da alteração citada (Pumphrey et al. 2013). Pode também acometer cães das raças Akita, Cocker Spaniel, Chow-Chow, Basset Hound, Beagle, Dachshund, Maltês, Pastor alemão, Pinscher, Schnauzer e, esporadicamente, cães mestiços (Reilly et al. 2005, Dubielzig et al. 2010, Scott et al. 2013).

Neste estudo, a avaliação histopatológica demonstrou que o glaucoma secundário foi o mais prevalente corroborando com as descrições de Gellat et al. (2007), Dubielzig et al. (2010) e Strom et al. (2011). Alterações intraoculares decorrentes de uveítes, endoftalmites e panoftalmites podem levar ao bloqueio do ângulo de filtração iridocorneal por células inflamatórias, hifemas, membranas fibrovasculares, sinéquias, cistos uveais, luxação de lente, ruptura de cápsula da lente e obliteração da malha trabecular por células contendo pigmento de melanina (Van Der Woerdt 2000, Gelatt \& Mackay 2004, Grahn \& Peiffer 2007, Tombran-Tink et al. 2008, Esson et al. 2009, Turner 2010, Alario et al. 2013, Scott et al. 2013).

Membranas fibrovasculares (MF), estruturas formadas por componentes fibrosos e vasculares (Dubielzig et al. 2010, Bauer et al. 2012) visualizadas em seis bulbos glaucomatosos deste estudo e cistos uveais, descritos como a separação entre as camadas epiteliais da úvea anterior (Deehr \& Dubielzig 1998), observados em quatro amostras, foram achados histopatológicos recorrentes. A membrana fibrovascular pode ocorrer nas porções anterior da íris (membrana fibrovascular pré-iridiana), posterior (membrana fibrovascular pós-iridiana) e recobrindo o corpo ciliar (membrana ciclítica) (Dubielzig et al. 2010, Sandberg et al. 2012). Embora os fatores que desencadeiam a formação da MF não estejam totalmente elucidados, Zarfoss et al. (2010) apontam que fatores angiogênicos como a liberação do fator de crescimento endotelial vascular em resposta a inflamações, neoplasias e hipóxia levam à sua formação. Como evidenciado neste estudo, a MF é um achado frequente em neoplasias, glaucomas e endoftalmites (Peiffer et al. 1990). A inversão ou eversão da porção pupilar da irís, respectivamente denominadas como entrópio e ectópio uveal, podem ocorrer em função da retração causada pela MF ou pelo encurvamento anterior ou posterior do músculo esfíncter da íris (Peiffer et al. 1990).

Cistos uveais em cães são considerados comuns e tem sido associado ao desenvolvimento de glaucoma nesta espécie (Deehr \& Dubielzig 1998, Gelatt \& Mackay 2004, Pumphrey et al. 2013). Geralmente são benignos, esféricos, podendo ser únicos ou múltiplos, uni ou bilaterais e encontrados na superfície do corpo ciliar e na região posterior da íris (Deehr \& Dubielzig 1998), corroborando com a literatura neste estudo tais cistos foram visualizados predominantemente na face posterior da íris em casos de glaucoma secundário.

Todas as amostras de bulbos oculares com apresentação clínica de quadros sugestivos de neoplasias enviadas para a análise anatomopatológica obtiveram a confirmação diagnóstica. No presente estudo, os neoplasmas ocorrerem em cães das raça Boxer (predisponente ao desenvolvimento de neoplasias intraoculares) (Turner 2010), Pastor Alemão, Cocker Spaniel, Beagle, Fila Brasileira e em um SRD.

Neste estudo, os neoplasmas de origem do epitélio iridociliar (dois carcinomas e um adenocarcinoma iridociliar) foram a principal neoplasia diagnosticada nos bulbos oculares de cães, embora Dubielzig et al. (2010) refiram-se a estes tumores como os de segunda maior ocorrência entre os cães. Eles surgem do epitélio do corpo ciliar e se estendem ao longo do corpo ciliar e da íris (Dubielzig et al. 2010, Labelle \& Labelle 2013), desencadeando sinais de uveíte, descolamento de retina e hemorragia intraocular (Dubielzig et al. 1998). O melanoma uveal, uma neoplasia melanocítica maligna primária, observada em apenas um Cocker Spaniel, não foi a neoplasia mais prevalente nos bulbos oculares avaliados. Apesar desse neoplasma ser considerado um dos mais frequentes nos olhos de cães (Esson et al. 2007, Grahn \& Sandmeyer 2007, Dubielzig et al. 2010), não é possível fazer especulações sobre sua frequência nos animais avaliados, devido ao número limitado de neoplasias oculares diagnosticadas.

Em gatos, foram diagnosticados dois carcinomas de células escamosas (CCE), e ambos exibiram proliferações neoplásicas originando-se na margem palpebral e invadindo o tecido corneal. Trata-se de uma neoplasia maligna localmente invasiva em felinos (Dubielzig et al. 2010, Scurrell et al. 2013), e de ocorrência comum em pálpebras (Slatter 2008, Scurrell 2013) e áreas pouco pigmentadas (Slatter 2008). A conjuntiva bulbar e os anexos oculares são normalmente 
afetados, sendo o acometimento da córnea geralmente secundário (Sandmeyer et al. 2008, Scurrell et al. 2013).

Embora não tenha sido um aspecto observado neste estudo, neoplasias intraoculares são apontadas como fatores predisponentes ao glaucoma secundário (Reilly et al. 2005, Tombran-Tink et al. 2008, Esson et al. 2009, Dubielzig et al. 2010, Yanoff \& Ducker 2014). Pode-se inferir que o glaucoma não foi desencadeado nos casos avaliados devido à detecção precoce dos tumores oculares, seguido pela enucleação.

Neste período, bulbos oculares submetidos à histopatologia decorriam de casos suspeitos de neoplasia, cuja remoção dos bulbos seguia-se à ultrassonografia ocular e à citologia. Atualmente, sabendo-se das possíveis complicações de olhos em Phthisis bulbi (Dubielzig et al. 2010) e da possibilidade do glaucoma primário apresentar-se bilateralmente (Reilly et al. 2005), imprimiu-se a obrigatoriedade da análise histopatológica nas condutas clínico-cirúrgicas de enucleações. Pode-se verificar que a perfuração ocular pode decorrer de afecções frequentes como a ceratoconjuntivite seca, de fácil diagnóstico e tratamento. 0 mesmo se verifica nos casos de glaucoma secundário, uma das mais importantes causas de cegueira e remoção do bulbo ocular em cães, decorrendo, na maioria das vezes, de causas inflamatórias (uveítes), muitas vezes responsivas ao tratamento quando precocemente diagnosticadas. Estas afecções, diferentemente das neoplasias oculares, podem ter sucesso terapêutico se adequadamente conduzidas. Desta forma, constata-se que há, ainda, grande necessidade de aperfeiçoamento na área da Oftalmologia Veterinária, a fim de se evitar condutas extremas como a perda ocular seguida da remoção do bulbo ocular de cães e gatos.

\section{CONCLUSÕES}

Constatou-se que as causas mais frequentes de exérese de bulbos oculares foram perfurações oculares, glaucoma e neoplasias.

A análise anatomopatológica confirmou as suspeitas de acometimento por neoplasias intraoculares observadas clinicamente, assim como os casos de glaucomas diagnosticados em primário e secundário, confirmando a importância dos exames histopatológicos na rotina oftalmológica.

Agradecimentos.- A todos os médicos veterinários que participaram do Serviço de Oftalmologia Veterinária (FAV-UnB) do LPV-UnB no referido período, bem como ao Laboratório de Histopatologia e Imunoistoquímica-Universidade Federal de Minas Gerais (UFMG).

\section{REFERÊNCIAS}

Alario A.F., Pizzirani S. \& Pirie C.G. 2013. Histopathologic evaluation of the anterior segment of eyes enucleated due to glaucoma secondary to primary lens displacement in 13 canine globes. Vet. Ophthalmol. 16:34-41.

Barachetti L., Rampazzo A., Mortellaro C.M., Scevola S. \& Gilger B.C. 2015. Use of episcleral cyclosporine implants in dogs with keratoconjunctivitis sicca: pilot study. Vet. Ophthalmol. 18:234-241.

Bauer B.S., Sandmeyer L.S., Hall R.B. \& Grahn B.H. 2012. Immunohistochemical evaluation of fibrovascular and cellular pre-iridal membranes in dogs. Vet. Ophthalmol. 15:54-59.

Deehr A.J. \& Dubielzig R.R. 1998. A histopathological study of iridociliary cysts and glaucoma in Golden Retrievers. Vet. Ophthalmol. 1:153-158.
Dubielzig R.R., Ketring K., McLellan G.J. \& Albert D.M. 2010. Veterinary Ocular Pathology: a comparative review. Saunders Elsevier, London. $456 \mathrm{p}$.

Dubielzig R.R., Steinberg H., Garvin H., Deehr A.J. \& Fischer B. 1998. Iridociliary epithelial tumors in 100 dogs and 17 cats: a morphological study. Vet. Ophthalmol. 1:223-231.

Esson D., Christin S., Fahre C.S., Zarfoss M.K. \& Dubielzig R.R. 2007. Suspected uveal metastasis of a nail bed melanoma in a dog. Vet. Ophthalmol. 10:262-266.

Esson D., Armour M., Mundy P., Schobert C.S. \& Dubielzig R.R. 2009. The histopathological and immunohistochemical characteristics of pigmentary and cystic glaucoma in the Golden Retriever. Vet. Ophthalmol. 12:361-368.

Gelatt K.N. \& Mackay E.O. 2004. Secondary glaucomas in the dog in North America. Vet. Ophthalmol. 7:245-259.

Gellat K.N., Brooks D.E. \& Kallberg M.E. 2007. The canine glaucomas, p.753-811. In: Gelatt K.N. (Ed.), Veterinary Ophthalmology. 4th ed. Blackwell, Oxford.

Grahn B.H. \& Peiffer R.L. 2007. Fundamentals of veterinary ophthalmic pathology, p.355-437. In: Gelatt K.N. (Ed.), Veterinary Ophthalmology. 4 th ed. Blackwell, Oxford.

Grahn B.H. \& Sandmeyer L.S. 2007. Diseases and surgery of the canine nasolacrimal system, p.637-655. In: Gelatt K.N. (Ed.), Veterinary ophthalmology. 4th ed. Blackwell, Oxford.

Hesse K.L., Fredo G., Guimarães L.L.B., Reis M.O., Pigatto J.A.T., Pavarini S.P., Driemeier D. \& Sonne L. 2015. Neoplasmas oculares e de anexos em cães e gatos no Rio Grande do Sul: 265 casos (2009-2014). Pesq. Vet. Bras. 3:49-54.

Labelle A.L. \& Labelle P. 2013. Canine ocular neoplasia: a review. Vet. Ophthalmol. 16:3-14.

Martins T.B. \& Barros C.S.L. 2014. Fifty years in the blink of an eye: a retrospective study of ocular and periocularlesions in domestic animals. Pesq. Vet. Bras. 34:1215-1222.

Massa K.L., Gilger B.C. \& Davidson M.G. 2002. Causes of uveitis in dogs: 102 cases (1989-2000). Vet. Ophthalmol. 5:93-98.

Matheis F.L., Walser-Reinhardt L. \& Spiess B.M. 2012. Canine neurogenic Keratoconjunctivitis sicca: 11 cases (2006-2010). Vet. Ophthalmol. 15:288-290.

McGavin M.D. \& Zachary J.F. 2009. Bases da Patologia em Veterinária. 4th ed. Elsevier, Rio de Janeiro. 1476p.

Paulsen M.E. \& Kass P.H. 2012. Traumatic corneal laceration with associated lens capsule disruption: a retrospective study of 77 clinical cases from 1999 to 2009. Vet. Ophthalmol. 15:355-368.

Peiffer Jr R.L., Wilcock B.P. \& Yin H. 1990. The pathogenesis and significance of pre-iridal fibrovascular membrane in domestic animals. Vet. Pathol. 27:41-45.

Pumphrey S.A., Pizzirani S., Pirie C.G. \& Needle D.B. 2013. Glaucoma associated with uveal cysts and goniodysgenesis in American Bulldogs: a case series. Vet. Ophthalmol. 16:377-385.

Reilly C.M., Morris R. \& Dubielzig R.R. 2005. Canine goniodysgenesis-related glaucoma: a morphologic review of 100 cases looking at inflammation and pigment dispersion. Vet. Ophthalmol. 8:253-258.

Salvio A.G. \& Marques M.E.A. 2006. Imuno-histoquímica para identificação de células neoplásicas no infiltrado ativo de melanomas finos. J. Bras. Patol. Med. Lab. 42:143-148.

Sandberg C.A., Herring I.P., Huckle W.R., Leroith T., Pickett, P. \& Rossmeisl J.H. 2012. Aqueous humor vascular endothelial growth factor in dogs: association with intraocular disease and the development of pre-iridal fibrovascular membrane. Vet. Ophthalmol. 15:21-30.

Sandmeyer L.S., Carrie B., Breaux C.B., Bruce H. \& Grahn B.H. 2008. Diagnostic ophthalmology. Can. Vet. J. 49:507-508.

Scott E.M., Esson D.W., Fritz K.J. \& Dubielzig R.R. 2013. Major breed distribution of canine patients enucleated or eviscerated due to glaucoma following routine cataract surgery as well as common histopathologic findings within enucleated globes. Vet. Ophthalmol. 16:64-72. 
Scurrell E.J., Lewin G., Solomons M. \& Rozmanec M. 2013. Corneolimbal squamous cell carcinoma with intraocular invasion in two cats. Vet. Ophthalmol. 16:151-154.

Slatter D. 2008. Fundamentals of veterinary ophthalmology. 4th ed. Saunders Elsevier. 478p.

Strom A.R., Hassig M., Iburg T.M. \& Spiess B.M. 2011. Epidemiology of canine glaucoma presented to University of Zurich from 1995 to 2009. 1. Congenital and Primary Glaucoma (4 and 123 cases). Vet. Ophthalmol. 14:121-126.

Tombran-Tink J., Barnstable C.J. \& Shields M.B. 2008. Mechanisms of the glaucomas. Humana Press, New Jersey. 747p.
Turner S.M. 2010. Oftalmologia em Pequenos Animais. Saunders Elsevier, Rio de Janeiro. 370p.

Van der Woerdt A. 2000. Lens-induced uveitis. Vet. Ophthalmol. 3:227-234.

Westermeyer H.D., Ward D.A. \& Abrams K. 2009. Breed predisposition to congenital alacrima in dogs. Vet. Ophthalmol. 12:1-5.

Yanoff M. \& Ducker J. 2014. Ophthalmology. 4th ed. Saunders Elsevier, China. $1733 \mathrm{p}$.

Zarfoss M.K., Breaux C.B., Whiteley H.E., Hamor R.E., Flaws J.A., Labelle P. \& Dubielzig R.R. 2010. Canine pre-iridal fibrovascular membranes: morphologic and immunohistochemical investigations. Vet. Ophthalmol. 13:4-13. 